\title{
RG stability of integrable fishnet models
}

\section{Ohad Mamroud and Genís Torrents}

Department of Particle Physics and Astrophysics, Weizmann Institute of Science, Rehovot, 7610001 Israel

E-mail: ohad.mamroud@weizmann.ac.il, genis.torrents@weizmann.ac.il

\begin{abstract}
We address the question of perturbative consistency in the scalar fishnet models presented by Caetano, Gürdoğan and Kazakov [1, 2]. We argue that their 3-dimensional $\phi^{6}$ fishnet model becomes perturbatively stable under renormalization in the large $N$ limit, in contrast to what happens in their 4-dimensional $\phi^{4}$ fishnet model, in which double trace terms are known to be generated by the RG flow. We point out that there is a direct way to modify this second theory that protects it from such corrections. Additionally, we observe that the 6-dimensional $\phi^{3}$ Lagrangian that spans an hexagonal integrable scalar fishnet is consistent at the perturbative level as well. The nontriviality and simplicity of this last model is illustrated by computing the anomalous dimensions of its $\operatorname{tr} \phi_{i} \phi_{j}$ operators to all perturbative orders.
\end{abstract}

Keywords: 1/N Expansion, Conformal Field Theory, Integrable Field Theories

ARXIV EPRINT: 1703.04152 


\section{Contents}

1 Introduction 1

2 Radiated loop corrections in regular fishnet models 5

3 Double traces for fishnet models $\quad 9$

$\begin{array}{lll}3.1 & \text { Fishnets within double trace corrected theories } & 10\end{array}$

3.2 Theories protected from double traces by additional flavour 11

4 Anomalous dimensions in the hexagonal fishnet model 12

4.1 Anomalous dimension of $\operatorname{tr} \phi_{1}^{2} \quad 13$

$\begin{array}{ll}4.2 \text { Anomalous dimension of } \operatorname{tr} \phi_{1} \phi_{2} & 15\end{array}$

$\begin{array}{lll}4.3 & \text { Integrability perspective } & 17\end{array}$

$\begin{array}{ll}\text { A Finite order checks for the re-summed result } & 19\end{array}$

$\begin{array}{lll}\text { A.1 Perturbative expansion of } \gamma_{11} & 19\end{array}$

$\begin{array}{lll}\text { A.2 } & \text { Perturbative expansion of } \gamma_{12} & 20\end{array}$

\section{Introduction}

For many decades, the inherent complexity to general nonperturbative QFT analysis has encouraged researchers to focus on two exceptionally convenient toy models: $\mathcal{N}=4 \mathrm{SYM}$ and ABJM. The study of these theories in the strong coupling regime makes use of two of their main virtues - their expected holographic realization [3] and their integrability in the 't Hooft limit [4]. Despite the lack of a formal proof for these properties, the amount of evidence supporting them both perturbatively and at the nonperturbative level is overwhelming. The abundance of nontrivial results that stems from the application of holographic and integrability techniques to these theories has located them, and especially $\mathcal{N}=4 \mathrm{SYM}$, in a privileged position as prospective departure points in the exploration of the nonperturbative landscape.

Recently, several efforts have been devoted to the characterization of the integrabilitypreserving continuous deformations of these maximally supersymmetric models [5-9], usually called $\gamma$-deformations in the literature. These type of deformations can be implemented in the holographic setting using a sequence of up to three independent T-duality, shift, Tduality (TsT) transformations. Equivalently, they are obtained in the field theoretic side by replacing the commutators of the theory with a Moyal $\star$-product with three twist parameters $\gamma_{i}$ directly related to the shifts in the holographic picture.

In this context an unexpected observation was made [1, 2]: a particular limit of $\gamma$ deformation that was dubbed the "double scaling limit" in [1] gives rise to scalar matrix models with classically marginal couplings and a surprisingly simple diagrammatic structure. More precisely, in the large $N$ limit of these models the only nontrivial dependence on the coupling for most quantities arises at the perturbative level from fishnet Feynman 
diagrams, i.e., arrangements of propagators in the shape of a regular lattice, which is made of triangular and square cells in the cases of ABJM and $\mathcal{N}=4$ SYM respectively. Both types of fishnet have been explicitly proven to be amenable to integrability [10], although in a framework that is not trivially connected to the inherited integrability from $\mathcal{N}=4$ SYM. The understanding on whether (or how) the two types of integrability are related could shed light on the field-theoretic origin of integrability in $\mathcal{N}=4$ SYM. In addition to this interest, the simplicity of the models raises hope that finite and strong coupling calculations could be carried out for them.

The aforementioned construction has, however, an important weakness: generic $\gamma$ deformations [11, 12], and in particular the fishnet model for the $\mathcal{N}=4 \gamma$-deformation [13], are unstable under RG flow, and therefore need to be corrected by adding double trace operators to the Lagrangian. Such terms are not unprecedented in deformations of $\mathcal{N}=4$ or ABJM: similar double trace corrections arise in the discrete deformations implemented by orbifolding the compact space in the holographic dual [14-17]. In the context of $A d S_{5}$ orbifolds it has been argued that their $\beta$ functions generically do not vanish on the real axis $[18,19]$, precluding the existence of a perturbative stabilization of the RG flow in any theory where neither these double trace corrections nor their flow are protected by additional symmetries. ${ }^{1}$

It remains unclear up to which point the RG flow stability condition can restrict analytically continued $\gamma$-deformations (for which unitarity has been relaxed): one might expect in these cases the possibility to fine tune the complex coupling to double trace operators at any perturbative order [13]. Be that as it may, in a stable theory defined by a double scaling limit the presence of such double trace elements at planar level significantly convulses the full diagrammatic of the problem, and restricts the applicability of integrability results to a narrow set of observables. Allegedly, one can still obtain useful results [1] for this protected sector. Nevertheless, many of the prospective applications of the fishnet models, whose interest may reach beyond the scope of $\gamma$ deformations due to their perturbative simplicity and nontriviality, disappear as soon as additional couplings are turned on in the Lagrangian.

In this note we present three results that show how this difficulty can be circumvented. Our first contribution in this regard is to prove that the fishnet model that stems out of ABJM is not afflicted with such perturbative counterterms in the large $N$ limit. In addition, we introduce a modification in the corresponding limit of $\mathcal{N}=4$ that can preclude this class of terms as well. Separately, we consider the set of hexagonal fishnet diagrams, which are the only class of integrable fishnet diagrams presented in [10] for which a fishnet theory had not been implemented yet. We present a 6-dimensional Lagrangian that produces this hexagonal theory, and prove its perturbative renormalizability at the planar limit.

Most of these results follow directly from the analysis of section 2, where each fishnet model with a regular lattice is considered separately in order to explicitly identify all its

\footnotetext{
${ }^{1}$ To the knowledge of the authors, only two examples are known to be free from such corrections: the supersymmetric $\gamma$ deformation, known as $\beta$-deformation [5], which implements a particular Leigh-Strassler deformation of $\mathcal{N}=4$ where the double trace operators do not flow, and the orbifold of type 0B suggested by Pedro Liendo, which is free from this type of operators by construction [20].
} 
possible RG flow instabilities. For the sake of generality, our analysis has not been restricted to scalar models; we allow for fermionic lines as well. Among the considered models, the triangular and hexagonal models with scalar fields are singled out as the only examples where double trace corrections are not turned on by loop corrections. In fact, the hexagonal model would also radiate $\operatorname{tr}^{2} \phi$ counterterms, but when this model is realized as a double scaling limit of a gauge theory these double trace terms are prevented by Gauss's law.

Section 3 focuses on double trace operators. After briefly discussing which observables would be sensitive to their presence at the planar limit provided a hypothetical RG stabilization for them was found, we switch to a suggestive observation: simple deformations of the scalar square fishnet model can perturbatively elude radiating double trace terms by means of a super-selection process that we call "refinement". It is implemented by equipping each type of field with an additional flavour index and making the insertions of interacting vertices shift this index cyclically. Despite reducing the amount of nontrivial observables and the set of orders at which they receive corrections, all the diagrams that contribute to the amplitudes of interest of the refined theory will still be governed by square fishnets.

The paper concludes with section 4 which illustrates the strong implications of the diagrammatic simplicity arising in these models: without even relying on integrability techniques, it is possible to re-sum the perturbative expansion for the anomalous dimension of $\operatorname{tr} \phi_{i} \phi_{j}$ to all orders. The re-summation matches the predictions at finite order, which are discussed in the appendix to the paper. The results are potentially useful from the integrability viewpoint. As we explain in section 4.3, our re-summation procedure has a natural interpretation from the perspective of [10], but computes quantities that involve nontrivial finite size corrections in the planar limit [21].

Figure 1 displays the branches of solutions for $\operatorname{tr} \phi_{i}^{2}$ and $\operatorname{tr} \phi_{i} \phi_{j \neq i}$ that are continuously connected to their classical values. Interestingly, there is a parametric window,

$$
\alpha \in\left(-\frac{3}{4} \sqrt{\frac{3}{19-18 C}}, \frac{9}{16}\right)
$$

where $C$ denotes the Catalan constant, in which both real branches simultaneously exist. The endpoints of these branches correspond to points where two real branches of solutions collide with each other. Speculatively, they could signal level crossings, instabilities or phase transitions for the system, despite there is a priori no reason to discard the possibility that these points lie already beyond the validity regime of our analysis because of other phase transitions or instabilities. In fact, it is tantalizing to attribute the three endpoints with $\gamma=-1$ to the fact that the square of the corresponding operator (which is of double trace type and irrelevant at $\alpha=0$ ) would become marginal with this anomalous dimension. Conversely, the presence of complex solutions for $\gamma$ beyond those points does not necessarily lead to inconsistencies. To the knowledge of the authors no principle is known which protects the anomalous dimensions from becoming complex in nonunitary theories.

In view of the RG stability of the examples considered along the paper, and of the finiteness and reality of the anomalous dimension computed in its last section, it is plausible to expect that some of the models under scrutiny have a well-defined nonperturbative 


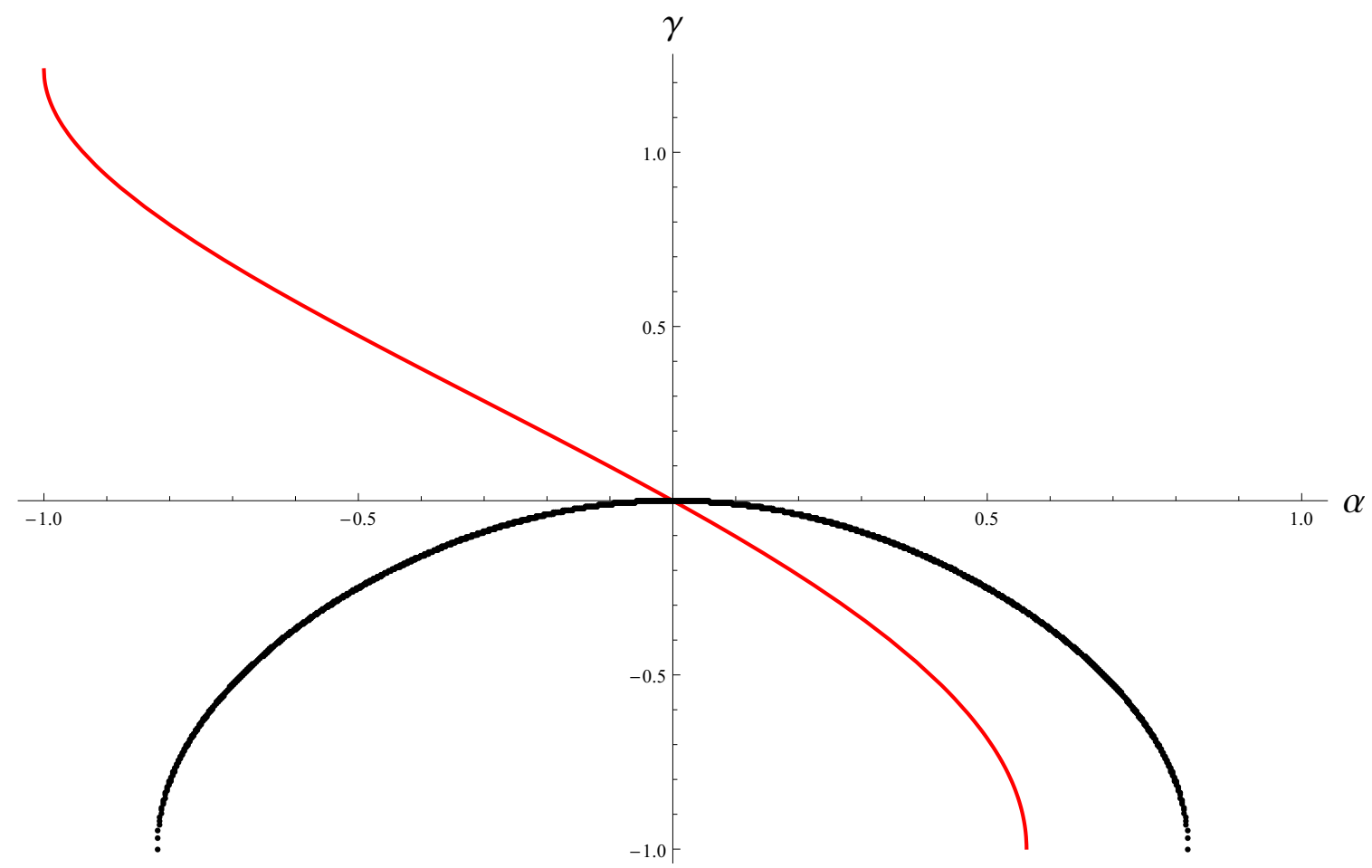

Figure 1. Anomalous dimension of $\operatorname{tr} \phi_{i}^{2}$ (black) and $\operatorname{tr} \phi_{i} \phi_{j \neq i}$ (red) in the hexagonal fishnet model as a function of the only meaningful combination of the couplings, $\alpha$.

completion. Since their actions are complex, it is natural to attempt such completion by taking the defining path integral of the theory as an integration over cycles given by Lefschetz thimbles. This framework has been shown in the literature to provide other complex theories with well-defined meanings: it links the stokes behaviour in the complexification of couplings to the accumulation of Yang-Lee zeroes in transition phenomena [22, 23], and potentially solves the ambiguity and ill-definiteness on theories with potentials unbounded from below [24]. In fact, this framework would constitute the appropriate setting to clarify the meaning of the different branches of solutions we find for $\gamma$, and understand what happens when they meet and whether it signifies a mixing with another operator. The authors plan to work on this question in the near future.

There are several other ways in which the analysis of the present paper can be extended: two natural questions are whether the integrability presented in [10] can be applied to other cases with fishnet structure, and whether the diagrammatic simplicity of the models considered allows for any simplification beyond planar level. In addition, it would be very interesting to clarify whether the proposed hexagonal and refined square fishnet models can be implemented as an integrability-preserving double scaling limit of a supersymmetric construction, and, if that is the case, how is the presence of double trace corrections avoided in them and what is their holographic description. 

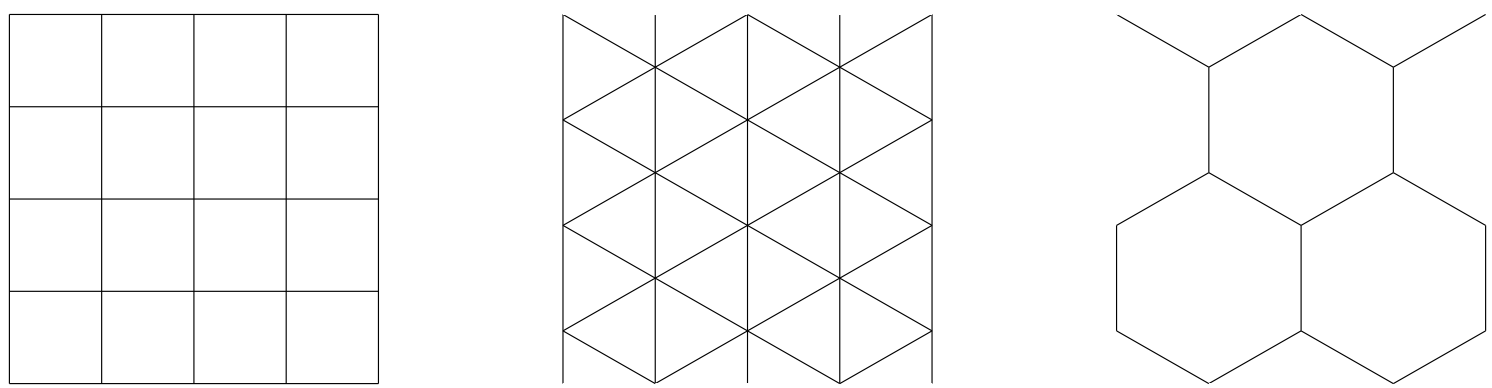

Figure 2. The three types of fishnets that have been proven integrable for, respectively, 4,3 and 6 dimensional target spaces happen to coincide with the three regular tilings of flat 2-dimensional space.

\section{Radiated loop corrections in regular fishnet models}

In this section we generalize the set of Lagrangians presented by Caetano, Gürdoğan and Kazakov [1,2] and discuss under which circumstances the resulting construction is stable under the RG flow, and therefore protected from the appearance of double trace operators.

The fishnet models are named that way because in the computation of the anomalous dimension of their single trace operators only a particular set of planar Feynman diagrams contribute: those that look like periodic planar lattices. For the sake of definiteness, in what follows we will restrict ourselves to the cases where these lattices are regular tessellations of the plane, of the types shown in figure 2. In fact, this particular case of diagrams are known to be amenable to integrable methods when all their lines are scalar [10], but even when that is not the case the simplicity of the perturbative expansion of the theory makes it a convenient toy model for perturbative problems and, plausibly, for resurgent methods.

If we do not take into account the need for renormalizability it is straightforward to engineer Lagrangians that produce only the desired planar fishnets. For the simple examples of figure 2, we proceed as follows: first, we associate a different species of field to every possible direction on the diagram. We choose these fields to be complex scalars or Dirac fermions in order to have oriented lines that distinguish in which vertex they begin and in which they end. In any case, we will suppose that these fields transform in the adjoint representation of some Lie group, and that this group has a well-defined large $N$ limit in which the algebra of fusion and fission of traces becomes that of $U(N)$, i.e.

$$
\left(T^{i}\right)_{b}^{a}\left(T_{i}\right)_{d}^{c} \propto \delta_{d}^{a} \delta_{b}^{c}+\ldots \rightarrow \begin{aligned}
& \operatorname{tr}\left(T^{i} A\right) \operatorname{tr}\left(T_{i} B\right) \propto \operatorname{tr}(A B)+\ldots \\
& \operatorname{tr}\left(T^{i} A T_{i} B\right) \propto \operatorname{tr}(A) \operatorname{tr}(B)+\ldots
\end{aligned}
$$

where the symbol "..." denotes terms that produce subleading contributions of order $\mathcal{O}\left(N^{-1}\right)$. The construction is completed by writing down interacting terms in the Lagrangian that mimic the chiral color ordering of the fishnet vertices with the chosen prescriptions. With this procedure, we obtain the following list $^{2}$ of fishnet candidates with

\footnotetext{
${ }^{2}$ The hexagonal fishnet theory with a fermionic species has not been included in the list, because it contains massless bosons, and therefore the tadpole diagrams that result from self-contractions are sensitive to the IR regulator.
} 
marginal interacting terms:

$$
\left\{\begin{array}{l}
\mathcal{S}_{+}^{\text {(proposed) }}=N \int d^{4} x \operatorname{Tr}\left(\frac{1}{2} \sum_{i=1}^{2}\left|\partial_{\mu} \phi^{i}\right|^{2}+\xi \phi_{1} \phi_{2} \phi_{1}^{\dagger} \phi_{2}^{\dagger}\right) \\
\mathcal{S}_{+, f}^{\text {(proposed) }}=N \int d^{3} x \operatorname{Tr}\left(\frac{1}{2}\left|\partial_{\mu} \phi\right|^{2}+\bar{\psi} \not \partial \psi+\xi \bar{\psi} \phi \psi \phi^{\dagger}\right) \\
\mathcal{S}_{+, 2 f}^{\text {(proposed) }}=N \int d^{2} x \operatorname{Tr}\left(\sum_{i=1}^{2} \bar{\psi}_{i} \not \partial \psi_{i}\right)+N \xi \bar{\psi}_{1} \bar{\psi}_{2} \psi_{1} \psi_{2} \\
\mathcal{S}_{*}^{\text {(proposed) }}=N \int d^{3} x \operatorname{Tr}\left(\frac{1}{2} \sum_{i=1}^{3}\left|\partial_{\mu} \phi^{i}\right|^{2}+\xi \phi_{1} \phi_{2} \phi_{3} \phi_{1}^{\dagger} \phi_{2}^{\dagger} \phi_{3}^{\dagger}\right) \\
\mathcal{S}_{\curlywedge \curlyvee}^{\text {(proposed) }}=N \int d^{6} x \operatorname{Tr}\left(\frac{1}{2} \sum_{i=1}^{3}\left|\partial_{\mu} \phi^{i}\right|^{2}+\xi_{1} \phi_{1}^{\dagger} \phi_{2} \phi_{3}+\xi_{2} \phi_{1} \phi_{2}^{\dagger} \phi_{3}^{\dagger}\right) \\
\mathcal{S}_{\curlywedge \curlyvee, 2 f}^{\text {(proposed) }}=N \int d^{4} x \operatorname{Tr}\left(\frac{1}{2}\left|\partial_{\mu} \phi^{i}\right|^{2}+\sum_{i=1}^{2} \bar{\psi}_{i} \not \partial \psi_{i}+\xi_{1} \bar{\psi}_{1} \psi_{2} \phi+\xi_{2} \bar{\psi}_{2} \phi \psi\right)
\end{array}\right\} .
$$

We shall remain unspecific in this paper about the spinor index structure. The upcoming discussion in this section is phrased in terms of dimensional analysis and the identification of planar diagrams, and therefore insensitive to this choice.

Most of the theories defined in (2.2) are not consistent as they stand: the proposed Lagrangians have not been written from the exhaustive list of relevant and marginal operators protected by some symmetry, and therefore it is plausible (and, indeed, the case) that the radiative corrections source other terms in the action along the RG flow. In order to identify which contribute at the planar level we need to focus on the set of superficially divergent planar diagrams and check (when their Feynman integral is divergent) their color structure.

Before we perform this task, however, it is convenient to emphasize the reasons underlying the diagrammatic simplicity of these models. Notice first that each interacting Lagrangian in (2.2) contains only single trace terms, and that in their entire sum each field and antifield appears only once. This uniquely fixes the way in which vertices can get contracted at the tree level. Moreover, it gives us access to the full list of connected diagrams, which can always be built from the appropriate tree via the contraction of a subset of its external legs.

This idea is of great help in the analysis of the large $N$ limit of the theory, when it is combined with the conventional $N$-power counting that follows from (2.1). When we draw a finite tree diagram on the plane with the proper color order of fields at each vertex, we have a cyclical ordered sequence of external legs along the perimeter of the figure, i.e., a single trace operator. Any conjugated pair in this sequence can be contracted, but, in doing so, as (2.1) indicates, the single trace is divided in two single trace factors. They correspond to the external legs found on either side of the line that represents the contraction. Further contractions within each factor are still possible at the planar level, 

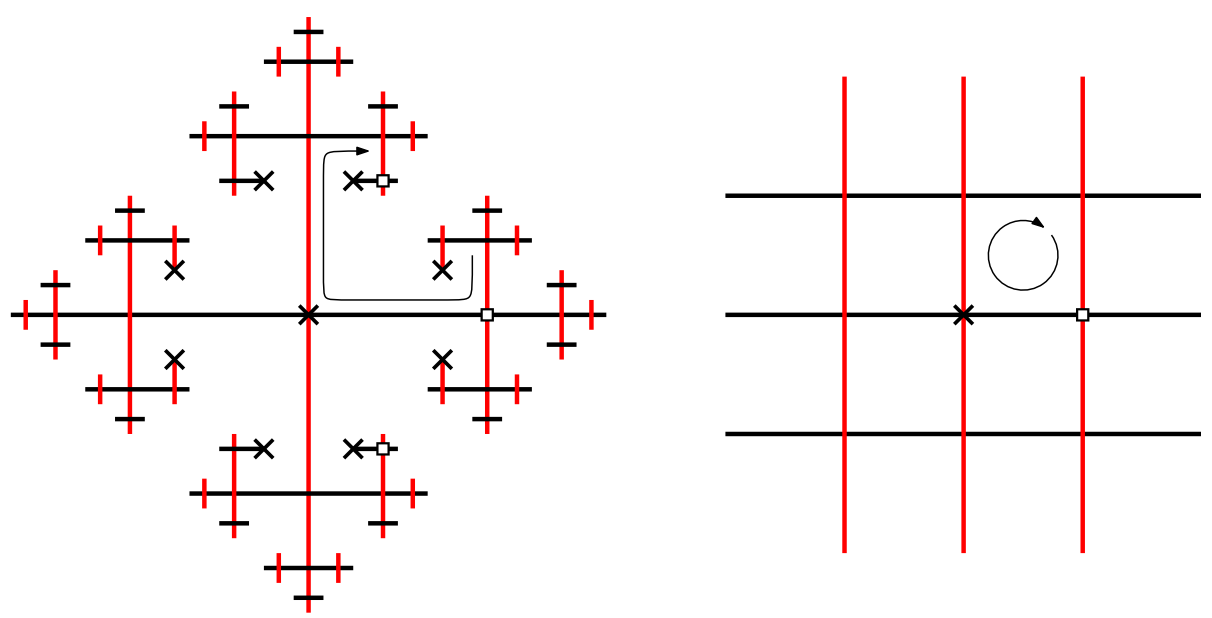

Figure 3. The tree structure for square fishnet models (left) can be depicted as a square fishnet (right). Horizontal lines represent one field species of field in the theory, vertical lines represent the other, we can consistently consider them all oriented rightwards and upwards. The nodes of the tree we have marked with the same symbol are represented by the same fishnet vertex. Wrapping once a tile of the fishnet corresponds to jumping from a branch of the tree to the consecutive one.

but any contraction between different blocks explicitly breaks the planarity of the diagram. As a consequence, when neighbouring contractions are not possible, every contraction will increase the amount of single trace factors in the color structure of the planar diagram. This fact importantly decimates the amount of planar diagrams we can construct with a single or double trace structure, and therefore it is a very convenient tool to discuss both perturbative divergences and single trace anomalous dimensions.

As an aside, let us mention that the graphic depictions of the tree diagram usually become impractical as their level increases due to the power growth in the number of legs and nodes at each level. It is convenient for our purposes to use a fishnet diagram to represent them. In this picture each vertex of the fishnet will represent different overlapped nodes of the tree in such a way that all overlapping legs are of the same type of field and the color structure is preserved in every vertex. ${ }^{3}$ Then, going once around a tile corresponds to jumping from a branch of the tree to the consecutive one. Figure 3 illustrates this construction for the square fishnet model. When we use this representation to depict the construction of generic planar diagrams, we notice that the single trace structures obtained after performing the simplest contractions are represented as closed paths (plausibly winding several times around different sets of tiles, see figure 4), on which we can perform additional contractions (see figure 5).

Equipped with our understanding of planar diagrams in fishnet models, we are now in position to discuss which among them can give rise to divergences. The simplest setting

\footnotetext{
${ }^{3}$ Since there is only a single vertex in the theory that can be added to every external line, we can construct the minimal fishnet that serves this purpose using the following procedure: for each external line, we add vertices to its closest neighbours in each side until the two closest legs are antifields of the original external leg. In the fishnet picture, the next vertex added to these neighbouring legs will overlap with the vertex from which the original leg emanates, so that the three legs of the same field are drawn on top of each other with consistent orientation.
} 

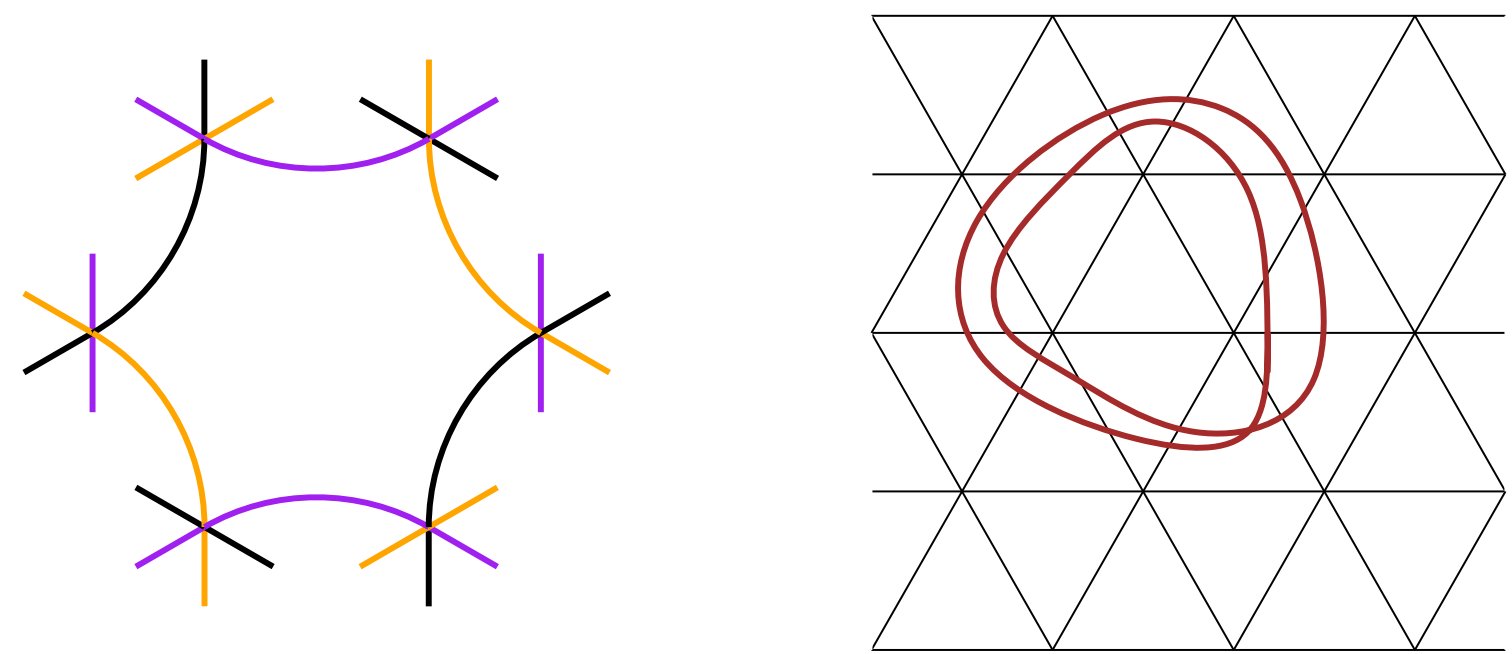

Figure 4. The hexagon diagram in the 3 dimensional fishnet model and the path on the lattice that produces it. External legs could be contracted with each other or with other tiles. Different fields in the diagram are painted with different colors. In the lattice, the type of filed is uniquely determined by the direction of the line.
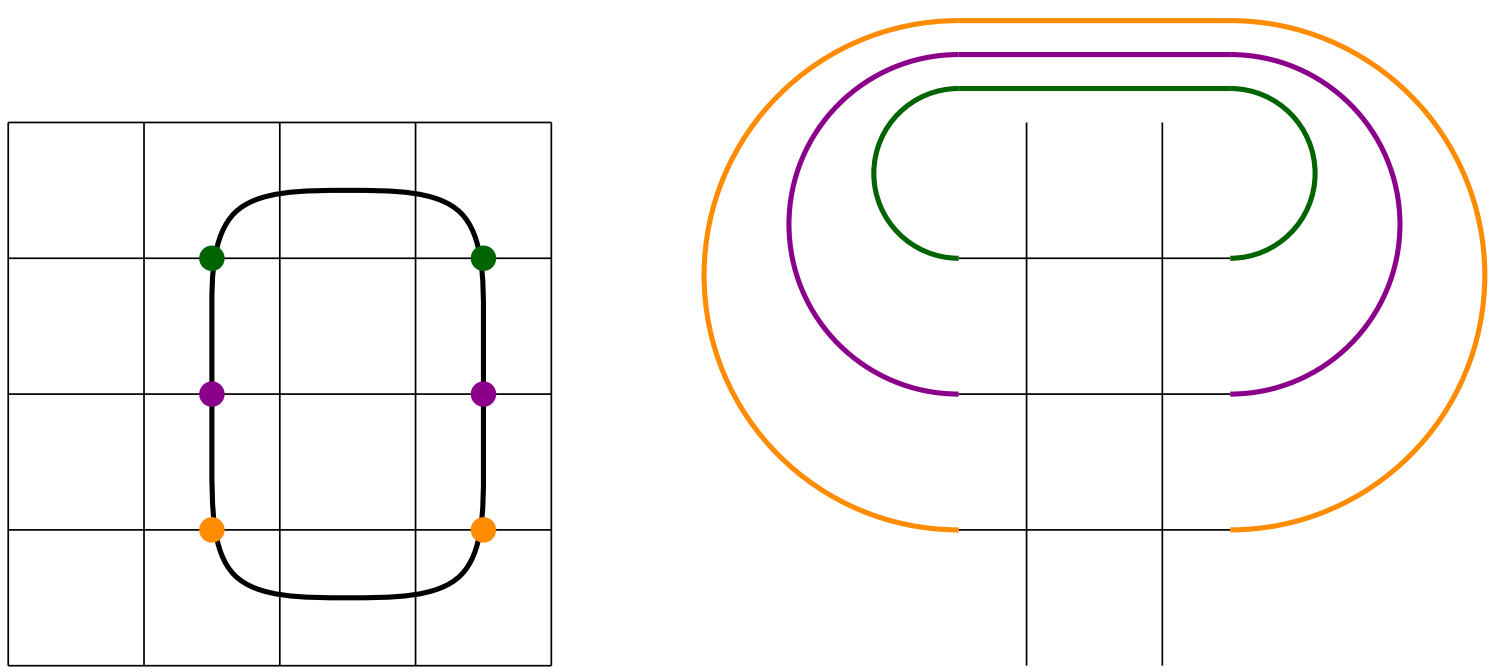

Figure 5. An example on how to transform a path in a lattice into a diagram. Points coloured with the same color are contracted afterwards. In the lattice, horizontal lines are $\phi_{1}$, vertical lines are $\phi_{2}$.

for addressing this question is dimensional regularization, in which contractions between a vertex and itself can be consistently put to zero. Let us examine each type of fishnet separately:

- Triangular lattice: as we go along a circuit, each external leg is separated from any leg that could potentially be contracted with it by at least four other legs. Notice that we exclude the possibility of self-contractions in this counting. No additional contraction can reduce the amount of legs between the contracted vertices below 4 . Therefore 


\begin{tabular}{|c|c|c|c|c|}
\hline Vertex & Lattice & D & Div. & Pathology \\
\hline $\operatorname{tr} \phi^{6}$ & Tri. & 3 & $3-\frac{1}{2} e_{\phi}$ & $\emptyset$ \\
\hline $\operatorname{tr} \phi^{4}$ & Sq. & 4 & $4-e_{\phi}$ & $\operatorname{tr}^{2} \phi^{2}$ \\
\hline $\operatorname{tr} \phi^{2} \psi^{2}$ & Sq. & 3 & $3-\frac{1}{2} e_{\phi}-e_{\psi}$ & $\operatorname{tr}^{2} \phi^{2}, \operatorname{tr}^{2}(\phi \psi)^{2}, \operatorname{tr}^{2} \phi^{3}$ \\
\hline $\operatorname{tr} \psi^{4}$ & Sq. & 2 & $2-\frac{1}{2} e_{\psi}$ & $\operatorname{tr}^{2} \psi^{2}$ \\
\hline $\operatorname{tr} \phi^{3}$ & Hex. & 6 & $6-2 e_{\phi}$ & $\left(\operatorname{tr}^{2} \phi\right)$ \\
\hline $\operatorname{tr} \phi \psi^{2}$ & Hex. & 4 & $4-e_{\phi}-\frac{3}{2} e_{\psi}$ & $\left(\operatorname{tr}^{2} \phi\right), \operatorname{tr}^{2} \phi^{2}$ \\
\hline
\end{tabular}

Table 1. For each theory in 2.2 here the schematic form of the vertex, its dimension, the superficial degree of divergence of its diagrams with $e_{\phi}$ external bosons and $e_{\psi}$ external fermions. The last column lists the divergent diagrams present in the perturbative expansion. Since none of the divergences in the list can be absorbed by the counterterms of the Lagrangian, only semisimple $\phi^{3}$ theories (when the Gauss's law is imposed on external states) and $\phi^{6}$ theories are immune to the radiation of additional terms in the Lagrangian.

the minimal amount of external legs on each side of the contraction on the path is 4 , and there are at least 8 external legs for each diagram with loops. As a result, the 3 dimensional theory introduced by [2] is conformal, at least perturbatively, in the planar limit.

- Hexagonal lattice: the minimal amount of external legs on each side of a contraction of the path is 1 . However, traces of single fields vanish in semisimple theories when the Gauss' law is imposed to external states. As a result, for the scalar theory in 6 dimensions there is no divergent diagram.

- Square lattice: the minimal amount of external legs on each side of a contraction of the path is 2 , and indeed the presence of loops with four external lines radiates double trace operators of the field squared in all theories of this fishnet type.

The list of potentially divergent diagrams in each of the theories of (2.2) is summarized in table 1. Figure 6 illustrates the simplest forms in which $\operatorname{tr}^{2} \phi$ and $\operatorname{tr}^{2} \phi^{2}$ divergences can appear.

\section{Double traces for fishnet models}

Double trace operators are a central piece of the puzzle of integrability-preserving deformations of $\mathcal{N}=4 \mathrm{SYM}$ and ABJM in absence of supersymmetry. The stabilization of their RG flow is the main difficulty in the construction of these theories. In this section we will briefly comment on the implications of their presence in models that would otherwise be of the "fishnet" type. Additionally, we will introduce a type of deformation for fishnet candidate Lagrangians, refinement, which protects them from developing double trace contributions. 


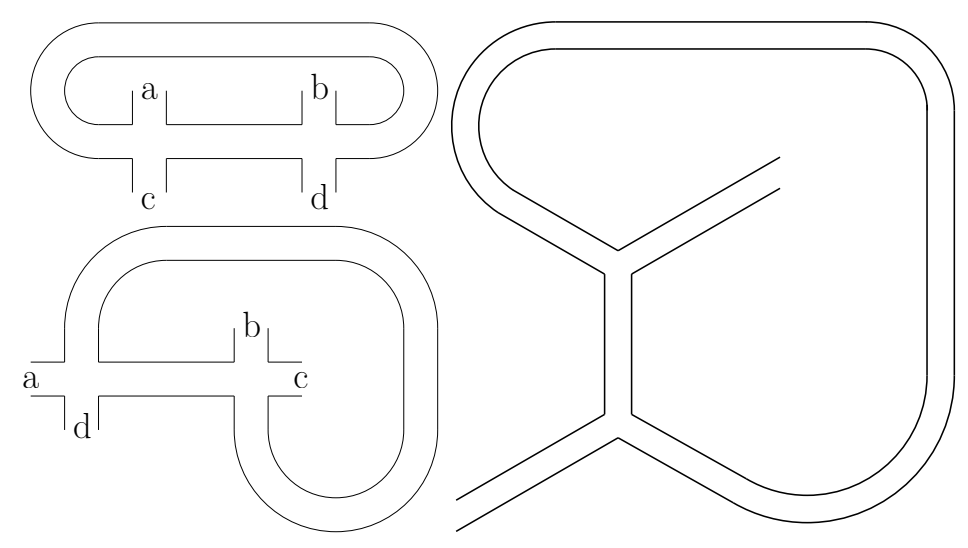

Figure 6. Candidate planar divergences in the square lattice models (left) and single trace divergence arising in the hexagonal lattice model (right).

\subsection{Fishnets within double trace corrected theories}

Most of the theories analysed in the previous section develop double trace operators under RG flow which break down the fishnet structure and in general render the model non-conformal. Indeed, both in the context of orbifolds $[16,17]$ and real gamma deformations [11], these terms rarely allow for perturbatively reachable stable fixed points: the zeroes of their one-loop beta functions generically lie in the imaginary axis of the complex plane. In fact, in a wide class of orbifolds, a no-go theorem precludes the existence of fixed points in nonsupersymmetric theories [18, 19].

When we relax the unitarity constraint, however, the restrictions on the availability of fixed points will not come from reality anymore. It is, to the knowledge of the authors, unclear whether additional constraints on the complexified parameter space will in general arise from demanding a consistent nonperturbative completion of the theory. However, at a speculative level, it was proposed in [13] to consider the scenario where appropriate fine-tuning of the complexified couplings of double trace terms allows us to perturbatively access a true stable fixed point of the theory under RG. Without committing ourselves to any conclusion on the viability of this construction, we will in what follows briefly analyse what observables in the theory are sensitive to the presence of double trace operators, and identify the sector left invariant by their presence.

A simple way to discuss the large $N$ behavior of diagrams with at least a double trace insertion it to observe what happens when this double trace insertion is replaced by a single trace vertex with the same external legs, see figure 7 . In regard to the 't Hooft limit counting it is not even necessary that such a single trace vertex is part of the theory. In the double line notation terminology, depending on whether the two line segments that get rearranged in the process were part or not of the same line, a loop is created or annihilated. Consequently, any double trace coupling with a vanishing $\beta$ function in the 't Hooft limit will appear suppressed by a $N^{-1}$ factor in the action, but contribute at the planar level to any diagram where the double trace insertion splits the color structure in two disconnected planar components. This $N^{-1}$ factor coincides with the one appearing in the 1-loop analysis of [13]. 

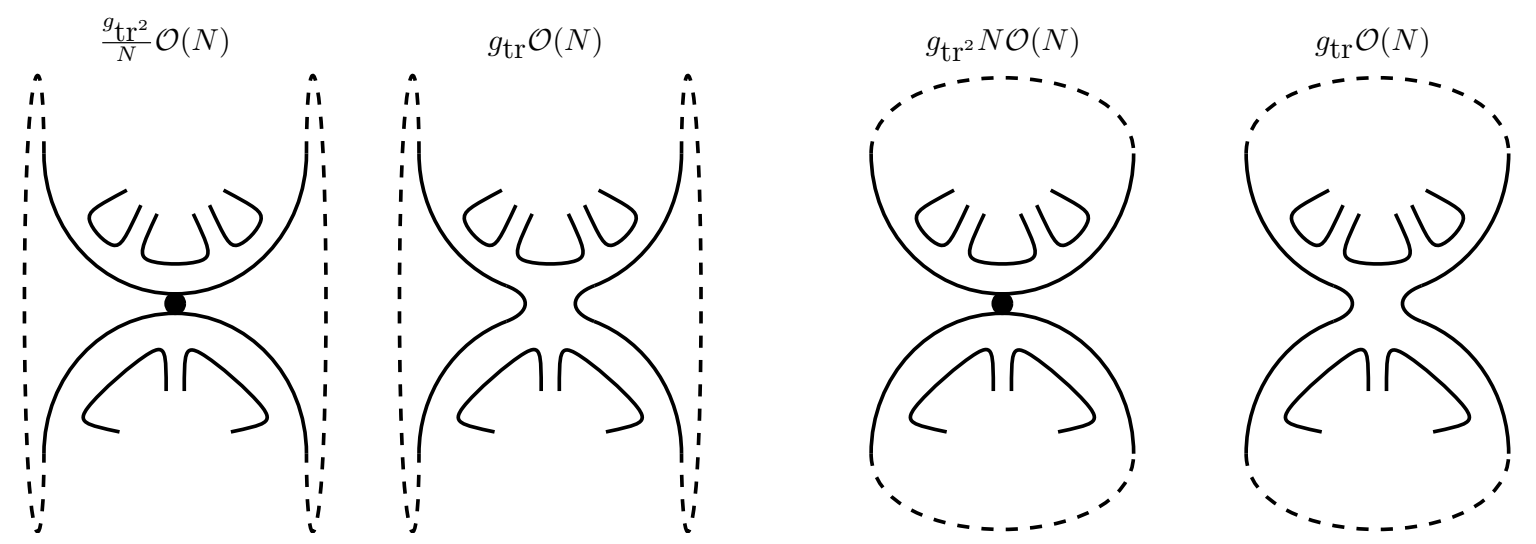

Figure 7. We depict in the double line notation the effect of replacing a generic double trace operator by a corresponding single trace construction, plausibly made out of several vertices. In the process the amount of loops either increases or decreases by a unit.

The functional formalism for field theory offers a particularly convenient way to construct a generating functional for the observables that remain unaffected by double traces at the planar level. From our previous discussion it should be clear that if double trace operators are the only possible cause for the splitting of the color double line diagram in disjoint components, they can only contribute at planar level when each of the double trace insertions causes an additional factorization of this type. Therefore, a Legendre transform with respect to bi-local sources, each one having its two positions coupled to the two traces in a specific double trace operator, will behave as the generating functional for the irreducible diagrams that stand protected from double trace planar corrections.

\subsection{Theories protected from double traces by additional flavour}

In the analysis of the perturbative sources of double trace operators performed in section 2 it became manifest that the addition of more vertices to a planar diagram would necessarily lead to an increase of the number of external legs. It is possible to take advantage of this fact when building models in order to completely deprive the double trace operators from entering the planar sector.

The simplest way to implement this is by a super-selection process that we shall denominate refinement: we add to each of the original species in the proposed action $\mathcal{S}^{\text {(proposed) }}$ up to two flavour indices in a specific manner that do not allow a simultaneous diagonalization of the free and interacting part of the Lagrangian. More precisely, we will choose in the diagonal basis for the free Lagrangian, an interaction that cyclically transits between flavours,

$$
\mathcal{L}_{i, j}^{(\mathrm{int})} \propto \delta_{i, j \bmod n+1} ; \quad i, j \in\{1, \ldots, n\} .
$$

In practice, this choice acts as a super-selection rule on the Feynman diagrams, that, provided the cycles are sufficiently large projects out all superficially divergent diagrams that could radiatively source double trace terms.

This strategy can be applied to the Gürdoğan-Kazakov square scalar fishnet candidate [1], as figure 8 illustrates. The resulting theory has three-indexed constituent scalar 

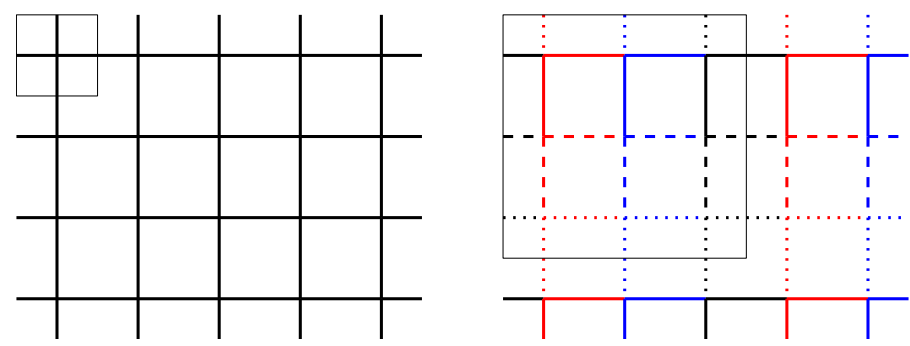

Figure 8. The refinement of the Gürdoğan-Kazakov's model with two flavour indices $a, b \in\{1,2,3\}$ super-selects from the fishnets of the original proposal (left) only those compatible with the resulting $3 \times 3$ color structure (right). The distinction is important when we study the diagrams which wrap a cylinder, such as those that compute anomalous dimensions of $\operatorname{tr} \phi^{L}$. The minimal periodic structure that allows to put the construction on the cylinder is in each case the unitary cell enclosed by a thin line.

fields $\phi_{i, a, b}$. One of the indices, $i \in\{1,2\}$, distinguishes their role in the fishnet structure; the other two, $a, b \in\{1,2,3\}$, are the indices introduced to refine the theory. The Lagrangian of the refined model reads

$$
\begin{aligned}
\mathcal{S}_{+}^{\text {(refined })} & =N \int d^{4} x \operatorname{Tr}\left(\frac{1}{2} \sum_{i=1, a, b=1}^{2,3}\left|\partial_{\mu} \phi_{a, b}^{i}\right|^{2}+\mathcal{L}_{\text {int }}\right) \\
\mathcal{L}_{\text {int }} & =\sum_{a, b=1}^{3} \xi_{a, b} \phi_{1, a, b} \phi_{2, a, b} \phi_{1, a_{\bmod 3}+1, b}^{\dagger} \phi_{2, a, b_{\bmod 3}+1}^{\dagger} \\
& =\sum_{a_{1}, a_{2}, b_{1}, b_{2}=1}^{3} \xi_{a_{1}, b_{1}} \phi_{1, a_{1}, b_{1}} \phi_{2, a_{1}, b_{1}} \phi_{1, a_{2}, b_{1}}^{\dagger} \phi_{2, a_{1}, b_{2}}^{\dagger} \delta_{a_{1}, a_{2} \bmod 3+1} \delta_{b_{1}, b_{2} \bmod 3+1}
\end{aligned}
$$

\section{Anomalous dimensions in the hexagonal fishnet model}

The diagrammatic simplicity of the fishnet models reveals itself in its full magnificence when we address the computation of anomalous dimensions of single trace operators. For them, the only allowed planar diagrams can be obtained by compactifying a periodic direction of the fishnet on a circle, plausibly with some shift, i.e., mixing the two translational symmetries of the unitary cell of the fishnet lattice. Not any cylindrical construction will be compatible with a given single trace operator, though: both the shift and the size of the compact direction $L$ in periodic unitary cells will be fixed uniquely by the given external operator. In contrast, the number of cells along the cylinder is not fixed and these operators develop a nontrivial dependence on the coupling.

The examples that conclude the present paper, namely the anomalous dimensions for the operators of $L=2$ in this model ( $\operatorname{tr} \phi_{i} \phi_{j}$ operators) to all orders, illustrate simultaneously the simplicity and nontriviality of the hexagonal fishnet model. For convenience, let 
us evoke the action of this theory, which was already presented in section 2 :

$$
\mathcal{S}_{\text {২Ү }}=N \int d^{D} x \operatorname{Tr}\left(\frac{1}{2} \sum_{i=1}^{3}\left|\partial_{\mu} \phi^{i}\right|^{2}+\xi_{1} \phi_{1}^{\dagger} \phi_{2} \phi_{3}+\xi_{2} \phi_{1} \phi_{2}^{\dagger} \phi_{3}^{\dagger}\right) .
$$

Here, $D$ will be taken to be $6-2 \epsilon$ in the setting of dimensional regularization. Notice that in this model the two vertices will appear alternately in any diagram. In practice, this implies that the perturbative expansion for any observable can be arranged in terms of the quantity

$$
\alpha \equiv \frac{\xi_{1} \xi_{2}}{(4 \pi)^{D / 2}} .
$$

The anomalous dimensions of $\operatorname{tr} \phi_{i} \phi_{j}$ operators in the hexagonal fishnet model can be computed at all perturbative orders via the resolution of a closed integral equation for a self-energy. The perturbative expansion up to an arbitrary order $n$ in $\alpha$ is also possible: the problem amounts to calculating the first $n$ terms in the series expansion of a known analytic function. The former method will be presented in this section. The comparison to the results of the latter method is described in detail in the appendix.

\subsection{Anomalous dimension of $\operatorname{tr} \phi_{1}^{2}$}

Let us first address the computation of the anomalous dimension of $\operatorname{tr} \phi_{1}^{2}$. Our theory is conformal and the operator under consideration does not undergo any mixing, so in dimensional regularization (with $D=6-2 \epsilon$ ) the relations between the bare and renormalized quantities of interest are

$$
\begin{aligned}
\alpha_{0} & =\mu^{2 \epsilon} \alpha \rightarrow \beta_{\alpha}=-2 \epsilon \alpha \\
\left(\operatorname{tr} \phi_{1}^{2}\right)_{0} & =\mathcal{Z}_{11}(\alpha) \operatorname{tr} \phi_{1}^{2} ; \quad \gamma_{11}=\frac{d \log \mathcal{Z}_{11}}{d \log \mu}=2 \epsilon \alpha \frac{d \log \mathcal{Z}_{11}^{-1}}{d \alpha} .
\end{aligned}
$$

A particular trait of our theory is the absence of field renormalization for the $\phi_{i}$ fields at the planar level. This allows us to read directly the anomalous dimension $\gamma_{11}$ from the renormalized correlator

$$
\Sigma_{11}(p) \equiv\left\langle\phi_{1}^{\dagger}(p) \phi_{1}^{\dagger}(-p) \operatorname{tr} \phi_{1}^{2}(0)\right\rangle_{1 \mathrm{PI}}=\mathcal{Z}_{11}^{-1} \cdot\left(\Sigma_{11}\right)_{0}
$$

where the subindex 1PI indicates that the correlator is 1 particle irreducible (with amputated external legs). From this point on, the conventional approach in perturbative analysis is to series expand $\left(\Sigma_{11}\right)_{0}$ in $\alpha$ and choose a prescription for the expansion

$$
\mathcal{Z}_{11}^{-1}=1+\sum_{n=1}^{\infty} \alpha^{n} z_{n}=1+\sum_{n=1}^{\infty} \alpha^{n} \sum_{m=0}^{n} \epsilon^{-m} a_{n, m}
$$

that renders the quantity $\Sigma_{11}$ finite. However, this particular problem permits a much more powerful approach that provides us with the re-summed quantity $\mathcal{Z}_{11}^{-1}$ to all perturbative orders. For this reason, the conventional perturbative analysis of this quantity has been relegated to the appendix, and in what follows we explain how this re-summed result 


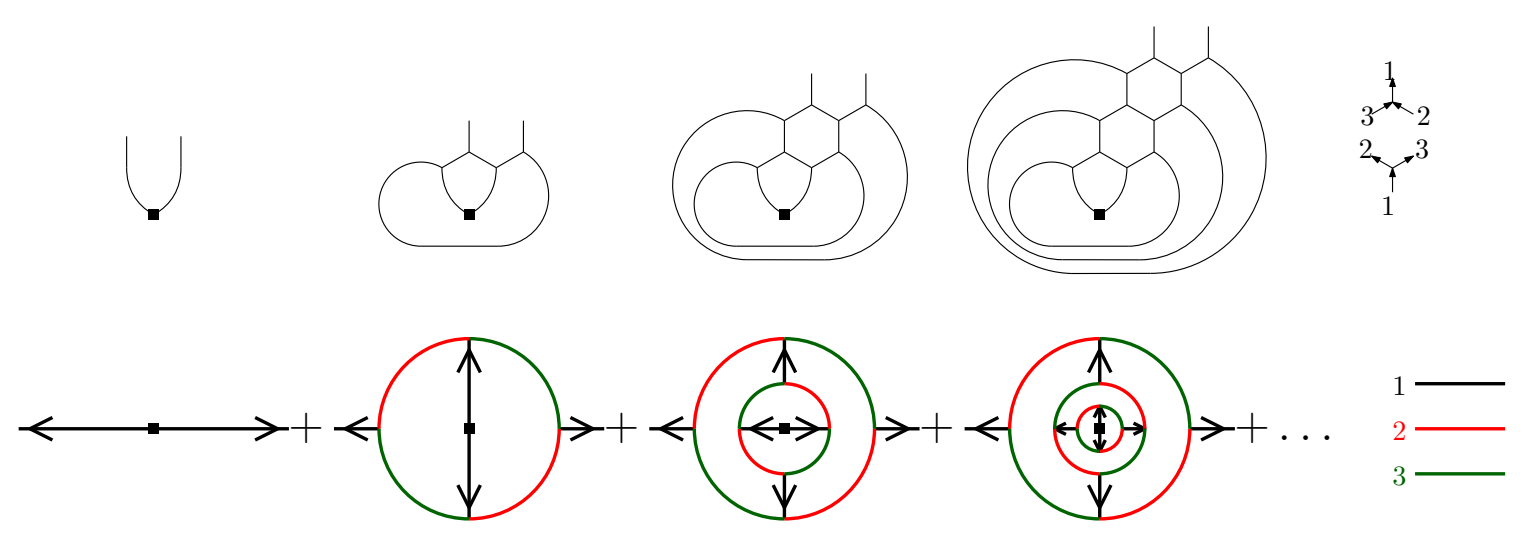

Figure 9. Diagrammatic expansion of $\Sigma_{11}$. Each color represents a different type of field, according to the legend on the right. The missing arrows can be inferred from the chirality of the vertices, they have been suppressed for the sake of clarity. The alternative representation of each diagram depicted above makes the fishnet structure explicit by associating a direction on the plane to each field.

is obtained. The concordance of the results of the two appraoches provides us with a nontrivial sanity check for the result.

The key point in the aforementioned re-summation is to notice that the perturbative expansion of $\Sigma_{11}$ has a nested structure, as depicted in figure 9 . This allows us to write the following integral equation:

$$
\Sigma_{11}(p)=\mathcal{Z}_{11}^{-1}+\alpha^{2} \pi^{2 \epsilon-6} \mu^{4 \epsilon} \int \frac{d^{6-2 \epsilon} q d^{6-2 \epsilon} r}{q^{2}(q+p)^{2} r^{2}(r+p)^{2}(q-r)^{4}} \Sigma_{11}(q-r) .
$$

The terms in the right hand side of this equation have divergences at $D \rightarrow 6$ that cancel among themselves. It is possible to sidestep this difficulty by applying the Laplacian operator to this equation. The resulting expression contains no divergence and therefore we can evaluate it at $\epsilon \rightarrow 0$ :

$$
\nabla^{2} \Sigma_{11}(p)=-4 \alpha^{2} \pi^{-6} \int \frac{d^{6} q d^{6} r}{q^{2}(q+p)^{4} r^{2}(r+p)^{4}(q-r)^{2}} \Sigma_{11}(q-r)
$$

Because $\Sigma$ is three point correlator of the conformal field theory when one of its momenta is put to zero, it can be shown it is of the form

$$
\Sigma_{11}=\sigma\left(\frac{\mu^{2}}{p^{2}}\right)^{\xi}
$$

where $\sigma$ denotes a constant that plausibly depends on $\alpha$. The relation between the anomalous dimension we want to compute and $\xi$ can be directly inferred from dimensional analysis in the Fourier transform of the three point function amplitude:

$$
\begin{aligned}
- \text { Power of } \mathrm{p} \text { in } \Sigma & =\# \text { integrals } \cdot D-\sum \Delta_{\text {free }}-\sum \gamma-\Delta_{\text {amputation }} \\
\xi & =\frac{2 D}{2}-\frac{4}{2} \Delta_{\phi}-\frac{1}{2} \gamma_{11}-\frac{2}{2} \Delta_{\phi}=-\frac{1}{2} \gamma_{11}
\end{aligned}
$$


All in all, we obtain a closed transcendental equation for $\gamma_{11}$ :

$$
\gamma_{11}\left(\gamma_{11}+4\right)=-4 \alpha^{2} \pi^{-6} \int \frac{d^{6} q d^{6} r}{q^{2}(q+p)^{4} r^{2}(r+p)^{4}(q-r)^{2-\gamma_{11}}}=-4 \alpha^{2} I_{2,2,1-\gamma_{11} / 2} .
$$

The function $I_{2,2,1-\gamma_{11} / 2}$ can be computed using an appropriate expansion in Gegenbauer polynomials [25] or inferred from recurrence relations [26]. In the notation of [26],

$$
\begin{aligned}
I_{a, b, c} & \equiv I_{a, b, c, a+b+c-D / 2} \equiv \pi^{-6} \int \frac{d^{6} q d^{6} r}{q^{2}(q+p)^{2 a} r^{2}(r+p)^{2 b}(q-r)^{2 c}} \\
(D-3) I_{a, b, c, d} & =b d G_{1, d+1}\left(G_{a, c+1} S_{D / 2-a-1, b-1, D / 2+a-d-2, d-b}+\{a \leftrightarrow b\}\right) \\
G_{x, y} & =\Gamma(D / 2-x) \Gamma(D / 2-y) \Gamma(x+y-D / 2) /(\Gamma(x) \Gamma(y) \Gamma(D-x-y)) \\
S_{a, b, c, d} & =\pi \cot (\pi c)\left(H_{a, b, c, d}\right)^{-1}-c^{-1}-\left(c^{-1}+d^{-1}\right) F_{a+c,-b,-c, b+d} \\
H_{a_{1}, a_{2}, a_{3}, a_{4}} & =\Gamma(1+a+b+c+d) \prod_{i=1}^{4} \Gamma\left(1+a_{i}\right) / \prod_{i, j=1}^{2} \Gamma\left(1+a_{i}+a_{2+j}\right) \\
F_{a, b, c, d} & ={ }_{3} F_{2}\left[\begin{array}{c}
-a-b 1 \\
1+c 1+d
\end{array} ;\right]-1
\end{aligned}
$$

Equation (4.10) can be resolved numerically, picking among the different branches of the solution the one that has the appropriate classical limit at $\alpha \rightarrow 0$. Figure 10 displays the results of this computation and compares it to the predictions of the leading perturbative terms computed in the appendix.

Notice the abrupt ending in a vertical slope of the physical branch of solutions to (4.10) at the points in which the anomalous dimension becomes -1 , namely at

$$
\alpha= \pm \frac{3}{4} \sqrt{\frac{3}{19-18 C}}
$$

where $C$ denotes the Catalan constant. At this point, this real branch of solutions folds back into another branch of the solution. The roots corresponding to these two branches become complex beyond this point of coalescence, and there is a priori no reason to identify one of them as the physically relevant one. As we discussed in the introduction, it is tempting to look for a physical interpretation of these breakdowns, but a meaningful discussion requires a better understanding of the complete spectrum or a nonperturbative formulation of the theory.

\subsection{Anomalous dimension of $\operatorname{tr} \phi_{1} \phi_{2}$}

The anomalous dimension of $\operatorname{tr} \phi_{1} \phi_{2}$ can be obtained using a procedure that, in form, is identical to the one we presented for $\operatorname{tr} \phi_{1}^{2}$. The diagrams are also organized in a nested structure, and all the discrepancies between the two cases are ultimately reduced to the different shape of the basic building block in this sequence, made explicit by the comparison of figure 11 to figure 9 .

The quantity of interest in this case is

$$
\Sigma_{12}(p) \equiv\left\langle\phi_{1}^{\dagger}(p) \phi_{2}^{\dagger}(-p) \operatorname{tr} \phi_{1} \phi_{2}(0)\right\rangle_{1 \mathrm{PI}}=\mathcal{Z}_{12}^{-1} \cdot\left(\Sigma_{12}\right)_{0}
$$




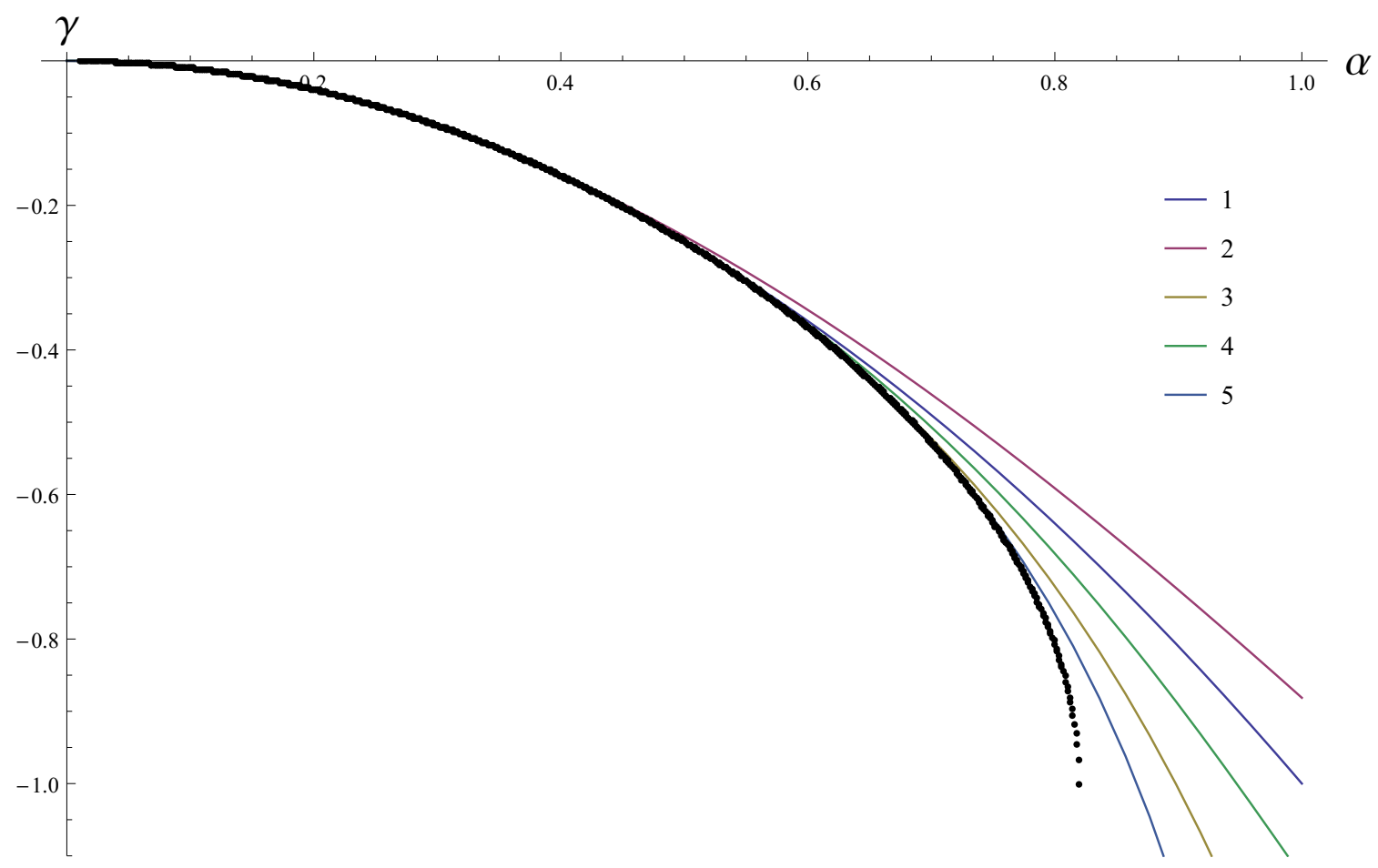

Figure 10. Convergence of the perturbative expansion to the exact result for the anomalous dimension $\gamma_{11}$. The thin lines correspond to truncated sums of the perturbative expansion at the orders indicated in the legend. The thick black dots are numerical evaluations of the re-summed result.

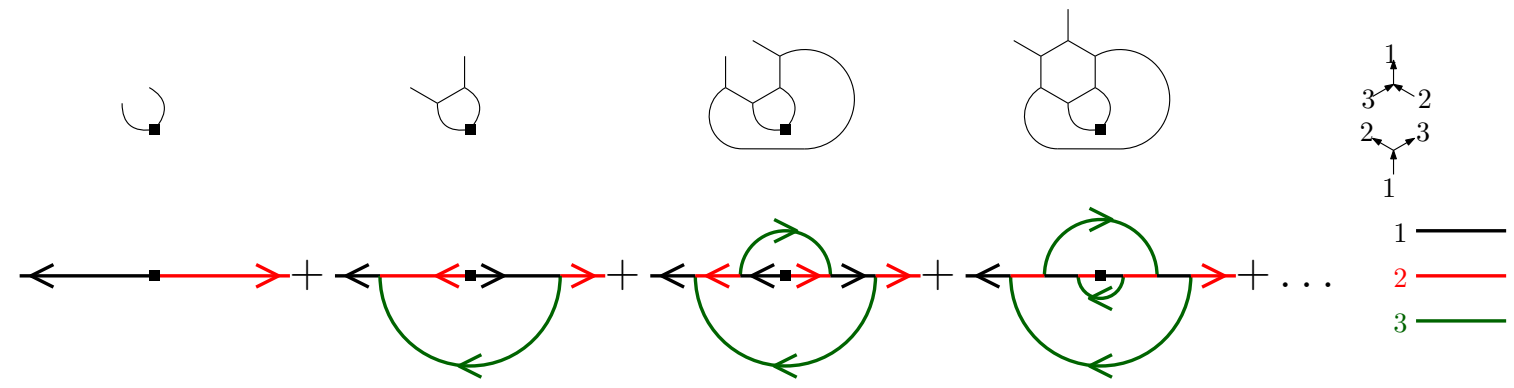

Figure 11. Diagramatic expansion of $\Sigma_{12}$. The conventions are identical to those of figure 9 .

By following the steps used in the computation of $\Sigma_{11}$ we now obtain

$$
\nabla^{2} \Sigma_{12}(p)=-4 \alpha \pi^{-3} \int \frac{d^{6} q}{q^{4}(p+q)^{4}} \Sigma_{12}(q)
$$

and again, we use the Ansatz

$$
\Sigma_{12}=\tilde{\sigma}\left(\frac{\mu^{2}}{p^{2}}\right)^{\xi}
$$

with $\xi=-\frac{1}{2} \gamma_{12}$. 


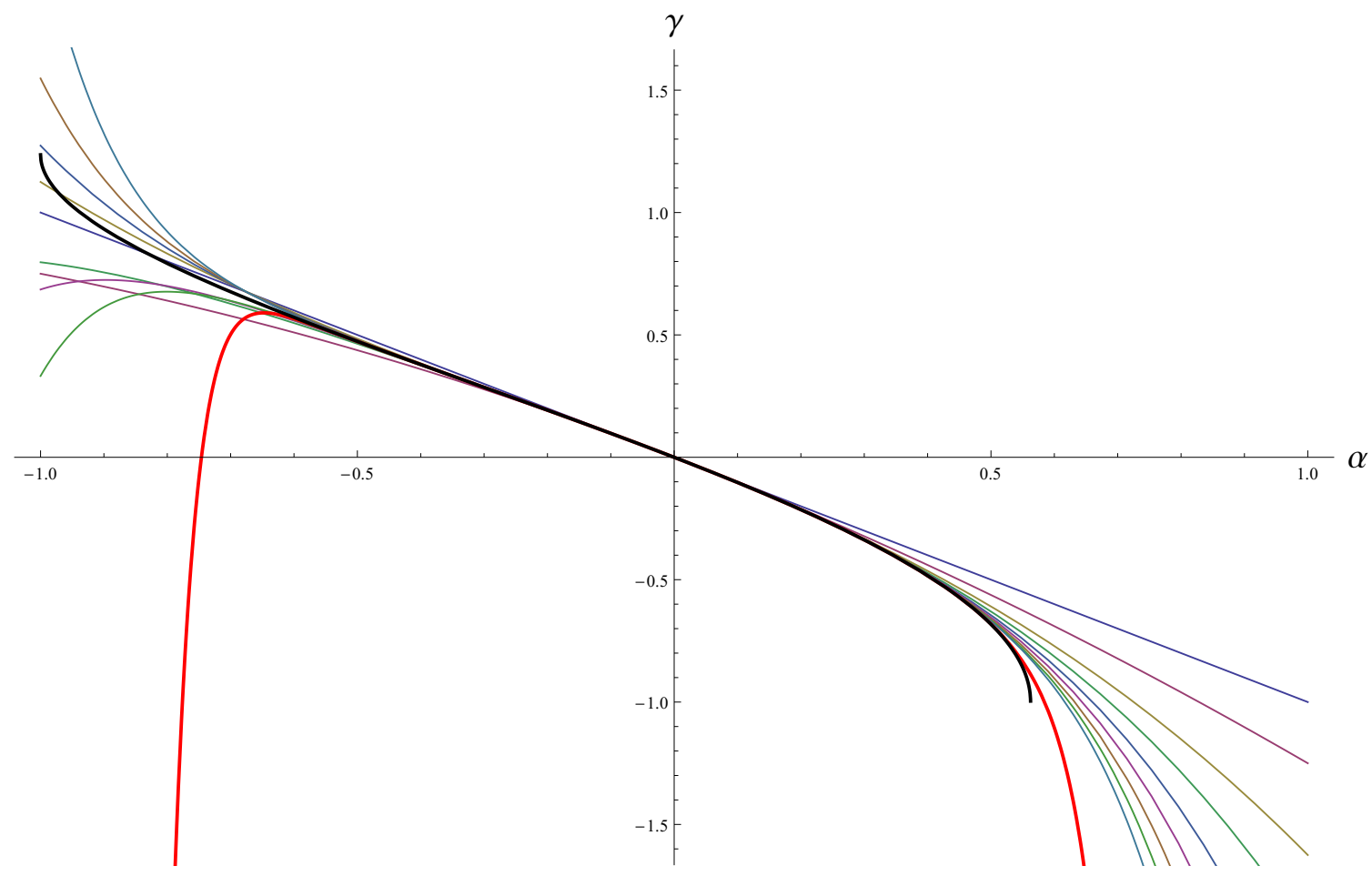

Figure 12. Convergence of the perturbative expansion to the exact result obtained for the anomalous dimension $\gamma_{12}$. The thin lines are truncated sums of the perturbative expansion at increasing orders. The red line is the 22 nd order, the highest the authors deemed appropriate to calculate. The thick black line is the exact result. Notice that the segment $\alpha \in(-1,-9 / 16)$ lies manifestly outside the radius of convergence of the perturbative series.

The solution here is much simpler than in the previous case, since the equation for the exponent becomes algebraic:

$$
\gamma(\gamma+4)=-4 \alpha \frac{\Gamma(1) \Gamma\left(1-\frac{\gamma}{2}\right) \Gamma\left(1+\frac{\gamma}{2}\right)}{\Gamma(2) \Gamma\left(2+\frac{\gamma}{2}\right) \Gamma\left(2-\frac{\gamma}{2}\right)}=\frac{-4 \alpha}{1-\left(\frac{\gamma}{2}\right)^{2}} .
$$

Among the four branches of solutions to this equation, given by $\gamma_{12}=-1 \pm \sqrt{5 \pm 4 \sqrt{1+\alpha}}$, the one that reaches the classical value at $\alpha \rightarrow 0$ is

$$
\gamma_{12}=-1+\sqrt{5-4 \sqrt{1+\alpha}}
$$

These results can be compared to the perturbative computation described in appendix A.2.

In contrast to what happens for $\gamma_{11}$, the behaviour of the anomalous dimension $\gamma_{12}$ is not symmetric in $\alpha$. Instead, it decreases monotonically between $\left(\alpha, \gamma_{12}\right)=(-1, \sqrt{5}-1)$ and $\left(\alpha, \gamma_{12}\right)=(9 / 16,-1)$, as it can be appreciated in figure 12. Both endpoints correspond to collisions of two real branches of solutions that become complex beyond them.

\subsection{Integrability perspective}

As an epilogue to this section let us briefly comment on the interest of the present models from the perspective of integrability. 
The organization of single trace two-point amplitudes in cylindrical diagrams of fixed radius allows for a translation of the problem into the language of $1+1$ spin chains, in close analogy to what happens in $\mathcal{N}=4 \mathrm{SYM}$ or ABJM for the chiral sector. The discussion on the integrability of these spin chain models can be addressed from two different perspectives. On the one hand, some fishnet models emerge as gamma deformations of $\mathcal{N}=4$ SYM and ABJM, and therefore, at least these cases are expected to share the same integrability properties, despite the fishnet case presents some structural differences in relation to the undeformed case. ${ }^{4}$ See [2] for a complete discussion on this integrability approach. On the other hand, as we mentioned in the introduction, Zamolodchikov proved in [10] that in scalar regular fishnet models the planar diagrams satisfy a Yang-Baxter equation in terms of their position/momentum propagators, which opens the perspective of applying integrability techniques of noncompact representations of orthogonal groups (see [27] and references therein, for instance). The simultaneous availability of these two types of integrability (one of which is strictly proven) makes this type of models an unparalleled probing ground to test integrability and understand it better.

The all-order results presented in this section can be viewed as the simplest implementation of Zamolodchikov's integrability, in the sense that ultimately our equations are reduced to an eigenvalue problem for the dilatation operator. From the spin chain perspective, nevertheless, the amplitudes computed correspond to operators with wrapping effects [21] of arbitrarily high order. Consequently, despite they are the simplest nontrivial operators one can compute in the theory, the authors expect that the results of this section can serve as a guiding principle when extending compact spin integrability results beyond the reach of Asymptotic Bethe Ansatz.

\section{Acknowledgments}

We would like to thank Vladimir Kazakov, Christoph Sieg, Matteo Bertolini and Zohar Komargodski for the critical reading of the manuscript. We also want to thank Amit Sever, Zohar Komargodski, Ran Yacoby, Masazumi Honda, Lorenzo Di Pietro, Mikhail Isachenkov and Vladimir Narovlansky for useful discussions. This work was supported in part by Israel Science Foundation (grant number 1989/14), by the ISF-UGC grant 1200/14 and by the ERC STG grant 335182. The research of GT is supported by a Koshland Postdoctoral fellowship, partially financed by the Koshland Fundation.

\footnotetext{
${ }^{4}$ Consider for instance, the operators with no shift in the cylinder, which are the fishnet correspondent to BMN vacua. In the fishnet theory, these observables are unprotected, and their anomalous dimension can be computed up to a finite order using the Y-system/Asymptotic Bethe Ansatz approach [1].
} 


$$
\stackrel{p}{\rightarrow} \int_{1}^{1} p=\int \frac{\mu^{2(6-D)} \lambda^{2} \tilde{\lambda}^{2} d^{D} q d^{D} r}{(2 \pi)^{2 D} q^{2}(q+p)^{2} r^{2}(r+p)^{2}(q-r)^{2 a}}=\alpha^{2}\left(\frac{p^{2}}{\mu^{2}}\right)^{D-4-a} I_{a}
$$

Figure 13. Elementary building block in the nested sequence depicted in figure 9 , and the value of its amputated Feynman integral. When the additional $\left(p^{2}\right)^{-2}$ power coming from the external legs is taken into account, a generic diagram of the sequence becomes a product of $I_{2+2 k \epsilon}$ factors in $D=6-2 \epsilon$ dimensions, with the corresponding $\alpha$ and $p^{2} / \mu^{2}$ powers.

\section{A Finite order checks for the re-summed result}

\section{A.1 Perturbative expansion of $\gamma_{11}$}

The perturbative expansion defined in figure 9 could be written as:

$$
\begin{aligned}
\Sigma_{11}(p) & =\mathcal{Z}_{11}^{-1}\left(1+\frac{\alpha^{2} \mu^{4 \epsilon}}{p^{2(2 \epsilon)}} I_{2}\left(1+\frac{\alpha^{2} \mu^{4 \epsilon}}{p^{2(2 \epsilon)}} I_{2+2 \epsilon}\left(1+\frac{\alpha^{2} \mu^{4 \epsilon}}{p^{2(2 \epsilon)}} I_{2+4 \epsilon}(1+\ldots)\right)\right)\right) \\
& =m^{2} \sum_{n=0}^{\infty} \alpha^{2 n} \sum_{\ell=0}^{n} z_{n-\ell}\left(\frac{p^{2}}{\mu^{2}}\right)^{-2 \epsilon \ell} \prod_{k=0}^{\ell-1} I_{2+2 k \epsilon}
\end{aligned}
$$

where $I_{a} \equiv I_{1,1, a}$ denotes the static factor in the nested structure in the Feynman diagrams as figure 13 illustrates, and we expanded $\mathcal{Z}_{11}$ according to the prescription of 4.5 .

The exact expression for $I_{\alpha}$ in dimensional regularization is simpler than the one for $I_{a, b, c}$, and was first derived in [28] using the uniqueness method [29], ${ }^{5}$ which leads to

$$
\begin{aligned}
& I_{a}=\frac{2 \Gamma\left(2-\frac{D}{2}\right) \Gamma\left(\frac{D}{2}-1\right)^{2} \Gamma(a+3-D) \Gamma\left(\frac{D}{2}-a-1\right)}{\Gamma(a)}\left(\frac{\Delta}{\Gamma\left(3-\frac{D}{2}\right) \Gamma\left(\frac{3 D}{2}-a-4\right)}-\frac{\Gamma(a) \cos \left(\pi\left(2-\frac{D}{2}\right)\right)}{\Gamma(D-2)}\right) \\
& \Delta=\frac{{ }_{3} F_{2}\left[\begin{array}{ccc}
1 & D-2 & a+2-\frac{D}{2} \\
3-\frac{D}{2} & a+3-\frac{D}{2}
\end{array} ;-1\right]}{a+2-\frac{D}{2}}+\frac{{ }_{3} F_{2}\left[\begin{array}{ccc}
1 & D-2 & D-a-2 \\
3-\frac{D}{2} & D-a-1
\end{array}-1\right]}{D-a-2} .
\end{aligned}
$$

The recent publication [31] presents new identities for hypergeometric functions that prove that A.2 is indeed equivalent to the expression that 4.11 provides for $I_{1,1, a}$.

Having at our disposal the explicit form of all elements in (A.1), we are now in position to series-expand them in $\epsilon$ in order to fix the counterterms $\delta_{m^{2}, n}$ and extract $\gamma_{\operatorname{tr} \phi^{2}}$ from them. Schematically, this expansion proceeds, for $D=6-2 \epsilon$, in the following terms:

$$
\begin{aligned}
I_{2+2 k \epsilon} & =\sum_{j=0}^{\infty} c_{k, j} \epsilon^{j-1} \\
z_{n} & =\sum_{k \leq n} a_{n, k} \epsilon^{-k} \\
\left(\frac{p^{2}}{\mu^{2}}\right)^{-2 \epsilon \ell} & =\sum_{n} \frac{(-2 \epsilon \ell)^{n}}{n !} \log ^{n}\left(\frac{p^{2}}{\mu^{2}}\right) .
\end{aligned}
$$

\footnotetext{
${ }^{5}$ See [30] for an updated review on this type of diagrams and other ways of obtaining this result.
} 
In the minimal subtraction scheme the $a_{n, k}$ coefficients are iteratively identified from the set of $c_{n, k}$, and a nontrivial sanity check on the computation is provided by the cancellation at every order of divergent terms carrying logarithms of the momentum by virtue of nondynamical counterterms fixed in previous orders. Let us for illustrative purposes explicit here the first few terms of this expansion:

$$
\begin{aligned}
& c_{k, 0}=\frac{1}{4(k+1)} ; \quad c_{k, 1}=\frac{13}{12}+\frac{7-4 \gamma_{E}}{8+8 k}-\zeta(3) ; \\
& c_{k, 2}=\frac{480\left(-3 k+3 \gamma_{E}-7\right)(k+1) \zeta(3)-12 \pi^{4}(k+1)-60 \gamma_{E}(26 k+47)+20 k(159 k+437)-30 \pi^{2}+360 \gamma_{E}^{2}+7135}{720(k+1)} ; \\
& a_{1,1}=-c_{0,0}=-\frac{1}{4} ; \quad a_{2,2}=\frac{c_{0,0}^{2}}{2}=\frac{1}{32} ; \quad a_{2,1}=\frac{c_{0,0}\left(c_{0,1}-2 c_{1,1}\right)}{2}=\frac{1}{8}\left(\zeta(3)-\frac{13}{12}\right) ; \\
& a_{3,3}=-\frac{c_{0,0}^{3}}{6} ; \quad a_{3,2}=-\frac{3 c_{0,0}\left(c_{0,1}-2 c_{1,1}\right)}{6} ; \quad a_{3,1}=-\frac{2 c_{0,0}\left(c_{0,1}-2 c_{1,1}\right)^{2}+c_{0,0}^{2}\left(c_{2,0}-4 c_{2,1}+3 c_{2,2}\right)}{6} ;
\end{aligned}
$$

Additional coefficients can be generated computationally. The authors have used the Mathematica package HypExp [32, 33] to obtain analytic expressions for $c_{k, n>0}$.

The RG flow equations (4.3) imply that we can read $\gamma_{11}$ from the $\epsilon^{-1}$ divergence in $\mathcal{Z}_{11}^{-1}$, by adding an additional factor of $2 n$ at order $\alpha^{n}$. The cancellation of higher order poles in $\epsilon$ has been verified numerically up to fifth order and provides a nontrivial check of the result for $I_{a}$. In addition, the perturbative expansion of the re-summed problem (obtained implicitly from equation (4.10) at $\alpha=0$ ):

$$
\gamma_{11}=\sum_{n=1}^{\infty} 4 n a_{n, 1} \alpha^{2 n}=-\alpha^{2}+\left(\zeta(3)-\frac{13}{12}\right) \alpha^{4}-\left(\zeta(3)^{2}+\frac{8}{3} \zeta(3)+\frac{41}{18}\right) \alpha^{6}+\ldots
$$

\section{A.2 Perturbative expansion of $\gamma_{12}$}

In the computation of the anomalous dimension of $\operatorname{tr} \phi_{1} \phi_{2}$ the elementary piece of the nested structure (figure 13) is replaced by the one represented in figure 14. Notice the change in the powers of $\alpha$ and $p^{2} / \mu^{2}$, and the replacement of $I_{2+2 k \epsilon}$ by $\tilde{I}_{2+k \epsilon}$, where the function $\tilde{I}$ is given by

$$
\tilde{I}_{a}=\frac{\Gamma\left(\frac{D}{2}-a\right) \Gamma\left(\frac{D}{2}-1\right) \Gamma\left(a+1-\frac{D}{2}\right)}{\Gamma(a) \Gamma(1) \Gamma(D-1-a)} .
$$

In order to keep the notation as parallel as possible, we expand

$$
\tilde{I}_{2+k \epsilon}=\sum_{j=0}^{\infty} \tilde{c}_{k, j} \epsilon^{j-1} .
$$

When we insert this expansion into

$$
\begin{aligned}
\Sigma_{12}(p) & =\mathcal{Z}_{12}^{-1}\left(1+\frac{\alpha \mu^{2 \epsilon}}{p^{2 \epsilon}} \tilde{I}_{2}\left(1+\frac{\alpha \mu^{2 \epsilon}}{p^{2 \epsilon}} \tilde{I}_{2+\epsilon}\left(1+\frac{\alpha \mu^{2 \epsilon}}{p^{2 \epsilon}} \tilde{I}_{2+2 \epsilon}(1+\ldots)\right)\right)\right) \\
& =\sum_{n=0}^{\infty} \alpha^{n} \sum_{\ell=0}^{n} \tilde{z}_{n-\ell}\left(\frac{p^{2}}{\mu^{2}}\right)^{-\epsilon \ell} \prod_{k=0}^{\ell-1} \tilde{I}_{2+k \epsilon}
\end{aligned}
$$




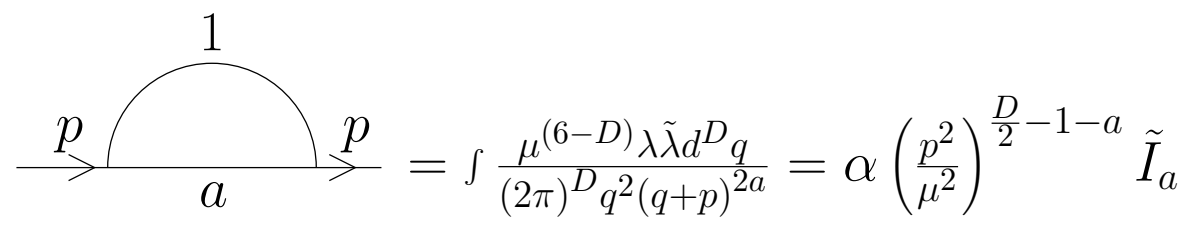

Figure 14. Elementary building block in the nested sequence depicted in figure 11, and value of its amputated Feynman integral. In this case the iterative factors of the sequence will be proportional to $I_{2+k \epsilon}$.

we notice that the relations between $a$ and $c$ can be directly extrapolated to $\tilde{a}$ and $\tilde{c}$. The values of $\tilde{c}$ themselves, in contrast, have to be computed from the new $\tilde{I}$ function:

$$
\tilde{c}_{k, 0}=\frac{1}{2(k+1)} ; \quad \tilde{c}_{k, 1}=\frac{k-2 \gamma_{E}+4}{4(k+1)} ; \quad \tilde{c}_{k, 2}=\frac{15 k^{2}-6 \gamma_{E} k+42 k-\pi^{2}+6 \gamma_{E}^{2}-24 \gamma_{E}+48}{24(k+1)} .
$$

We obtain

$$
\gamma_{12}=\sum_{n=1}^{\infty} 2 n \tilde{a}_{n, 1} \alpha^{n}=-\alpha-\frac{1}{4} \alpha^{2}-\frac{3}{8} \alpha^{3}-\ldots
$$

in concordance to the re-summed expression. The numerical verification has been performed up to 22 orders, see figure 12 .

Open Access. This article is distributed under the terms of the Creative Commons Attribution License (CC-BY 4.0), which permits any use, distribution and reproduction in any medium, provided the original author(s) and source are credited.

\section{References}

[1] Ö. Gürdoğan and V.A. Kazakov, New Integrable 4D Quantum Field Theories from Strongly Deformed Planar $\mathcal{N}=4$ Supersymmetric Yang-Mills Theory, Phys. Rev. Lett. 117 (2016) 201602 [Addendum ibid. 117 (2016) 259903] [arXiv: 1512.06704] [INSPIRE].

[2] J. Caetano, Ö. Gürdoğan and V.A. Kazakov, Chiral limit of $N=4 S Y M$ and ABJM and integrable Feynman graphs, arXiv: 1612.05895 [INSPIRE].

[3] O. Aharony, S.S. Gubser, J.M. Maldacena, H. Ooguri and Y. Oz, Large-N field theories, string theory and gravity, Phys. Rept. 323 (2000) 183 [hep-th/9905111] [INSPIRE].

[4] N. Beisert et al., Review of AdS/CFT Integrability: An Overview, Lett. Math. Phys. 99 (2012) 3 [arXiv: 1012.3982] [INSPIRE].

[5] O. Lunin and J.M. Maldacena, Deforming field theories with $\mathrm{U}(1) \times \mathrm{U}(1)$ global symmetry and their gravity duals, JHEP 05 (2005) 033 [hep-th/0502086] [INSPIRE].

[6] S. Frolov, Lax pair for strings in Lunin-Maldacena background, JHEP 05 (2005) 069 [hep-th/0503201] [INSPIRE].

[7] N. Beisert and R. Roiban, Beauty and the twist: The Bethe ansatz for twisted $N=4 S Y M$, JHEP 08 (2005) 039 [hep-th/0505187] [INSPIRE].

[8] H.-H. Chen, P. Liu and J.-B. Wu, Y-system for $\gamma$-deformed ABJM Theory, JHEP 03 (2017) 133 [arXiv: 1611.02804] [INSPIRE]. 
[9] E. Imeroni, On deformed gauge theories and their string/M-theory duals, JHEP 10 (2008) 026 [arXiv: 0808.1271] [INSPIRE].

[10] A.B. Zamolodchikov, "Fishing-net" diagrams as a completely integrable system, Phys. Lett. B 97 (1980) 63 [INSPIRE].

[11] J. Fokken, C. Sieg and M. Wilhelm, Non-conformality of $\gamma_{i}$-deformed $N=4 S Y M$ theory, $J$. Phys. A 47 (2014) 455401 [arXiv: 1308.4420] [inSPIRE].

[12] J. Fokken, C. Sieg and M. Wilhelm, A piece of cake: the ground-state energies in $\gamma_{i}$-deformed $\mathcal{N}=4$ SYM theory at leading wrapping order, JHEP 09 (2014) 078 [arXiv:1405.6712] [INSPIRE].

[13] C. Sieg and M. Wilhelm, On a CFT limit of planar $\gamma_{i}$-deformed $\mathcal{N}=4$ SYM theory, Phys. Lett. B 756 (2016) 118 [arXiv:1602.05817] [INSPIRE].

[14] S. Kachru and E. Silverstein, $4 D$ conformal theories and strings on orbifolds, Phys. Rev. Lett. 80 (1998) 4855 [hep-th/9802183] [INSPIRE].

[15] A.E. Lawrence, N. Nekrasov and C. Vafa, On conformal field theories in four-dimensions, Nucl. Phys. B 533 (1998) 199 [hep-th/9803015] [INSPIRE].

[16] M. Bershadsky, Z. Kakushadze and C. Vafa, String expansion as large- $N$ expansion of gauge theories, Nucl. Phys. B 523 (1998) 59 [hep-th/9803076] [inSPIRE].

[17] M. Bershadsky and A. Johansen, Large-N limit of orbifold field theories, Nucl. Phys. B 536 (1998) 141 [hep-th/9803249] [INSPIRE].

[18] A. Dymarsky, I.R. Klebanov and R. Roiban, Perturbative gauge theory and closed string tachyons, JHEP 11 (2005) 038 [hep-th/0509132] [INSPIRE].

[19] A. Dymarsky, I.R. Klebanov and R. Roiban, Perturbative search for fixed lines in large- $N$ gauge theories, JHEP 08 (2005) 011 [hep-th/0505099] [INSPIRE].

[20] P. Liendo, Orientifold daughter of $N=4$ SYM and double-trace running, Phys. Rev. D 86 (2012) 105032 [arXiv:1107.3125] [INSPIRE].

[21] C. Sieg and A. Torrielli, Wrapping interactions and the genus expansion of the 2-point function of composite operators, Nucl. Phys. B 723 (2005) 3 [hep-th/0505071] [INSPIRE].

[22] G. Guralnik and Z. Guralnik, Complexified path integrals and the phases of quantum field theory, Annals Phys. 325 (2010) 2486 [arXiv:0710.1256] [INSPIRE].

[23] T. Kanazawa and Y. Tanizaki, Structure of Lefschetz thimbles in simple fermionic systems, JHEP 03 (2015) 044 [arXiv: 1412.2802] [INSPIRE].

[24] E. Witten, Analytic Continuation Of Chern-Simons Theory, AMS/IP Stud. Adv. Math. 50 (2011) 347 [arXiv:1001.2933] [INSPIRE].

[25] A.V. Kotikov, The Gegenbauer polynomial technique: The Evaluation of a class of Feynman diagrams, Phys. Lett. B 375 (1996) 240 [hep-ph/9512270] [INSPIRE].

[26] D.J. Broadhurst, J.A. Gracey and D. Kreimer, Beyond the triangle and uniqueness relations: Nonzeta counterterms at large-N from positive knots, Z. Phys. C 75 (1997) 559 [hep-th/9607174] [INSPIRE].

[27] D. Chicherin, S. Derkachov and A.P. Isaev, Conformal group: R-matrix and star-triangle relation, JHEP 04 (2013) 020 [arXiv:1206.4150] [InSPIRE]. 
[28] D.I. Kazakov, The method of uniqueness, a new powerful technique for multiloop calculations, Phys. Lett. B 133 (1983) 406 [inSPIRE].

[29] D.I. Kazakov, Multiloop Calculations: Method of Uniqueness and Functional Equations, Theor. Math. Phys. 62 (1985) 84 [Teor. Mat. Fiz. 62 (1984) 127] [InSPIRE].

[30] A.G. Grozin, Massless two-loop self-energy diagram: Historical review, Int. J. Mod. Phys. A 27 (2012) 1230018 [arXiv: 1206 . 2572] [INSPIRE].

[31] A.V. Kotikov and S. Teber, New results for a two-loop massless propagator-type Feynman diagram, arXiv: 1611.07240 [INSPIRE].

[32] D. Maître, HPL, a mathematica implementation of the harmonic polylogarithms, Comput. Phys. Commun. 174 (2006) 222 [hep-ph/0507152] [INSPIRE].

[33] D. Maître, Extension of HPL to complex arguments, Comput. Phys. Commun. 183 (2012) 846 [hep-ph/0703052] [inSPIRE]. 